\title{
Qualité gustative et nutritionnelle des gruyères hyposodés
}

\author{
D. LEFIER *, R. GRAPPIN *, G. GROSCLAUDE ** et G. CURTAT *** \\ * INRA, Station Expérimentale Laitière, 39800 Poligny, France \\ ** INRA, Service d'Etude et de Réalisation Technologique, 44072 Nantes, France \\ *** Ets P. GRILLOT, 39800 Poligny, France
}

\section{Résumé}

Pour pallier le manque de saveur des fromages à pâte pressée cuite à faible teneur en chlorure de sodium, nous avons substitué des solutions riches en chlorure de magnésium issues de l'extraction du sel marin, au chlorure de sodium. Nous sommes ainsi parvenus à réduire de $80 \%$ la teneur en sodium des fromages $(50 \mathrm{mg}$ au lieu de $270 \mathrm{mg}$ pour $100 \mathrm{~g}$ de fromage) alors que la teneur en magnésium a doublé.

Cette diminution de la teneur en sodium des fromages a peu modifié les phénomènes fermentaires et protéolytiques au cours de l'affinage.

Sur le plan organoleptique, bien que se différenciant peu des fromages témoins, les fromages hyposodés présentaient une légère amertume et une pâte plus souple. De ce fait, ce gruyère à faible teneur en sodium permet d'élargir la gamme des fromages proposés aux consommateurs contraints à une alimentation hyposodée.

Mots clés: Gruyère de Comté - Fromage hyposodé - Qualité.

\section{Summary}

Sensory properties and nutritional quality of low-sodium Gruyère cheese

Because of a lack of flavour of low sodium, Gruyère-type cheese, a magnesium chloride solution was used instead of sodium chloride. Using this solution the residual sodium content of the cheese was lowered by $80 \%(50 \mathrm{mg}$ instead of $270 \mathrm{mg}$ for the control cheeses), and the magnesium content was increased by two fold. The amount of free fatty acids and degree of proteolysis after the ripening were similar to those of controls. In spite of a slight but significant bitterness, and a softer body, low sodiumcheeses were found quite acceptable by the taste panel. Consumption of Gruyère cheese salted with this mixture, appears to be a good alternative for people who are on low-sodium diet.

Key words : Gruyère of Comté - Low-sodium cheese - Quality. 


\section{Introduction}

On admet généralement qu'il existe une relation entre l'ingestion de sodium et la fréquence d'anomalies cardio-vasculaires comme l'hypertension artérielle.

Cet état de fait a incité les chercheurs et les industriels à mettre en œuvre des moyens permettant de réduire la teneur en sodium notamment dans le cas des produits laitiers, tout en essayant de préserver les caractéristiques organoleptiques originelles.

Dans ce but, diverses voies ont été testées, comme la suppression du salage des fromages (ThaKur et al., 1974; CurTat, 1982), la réduction du temps de saumurage (Delbeke et al., 1982) ou encore la substitution du chlorure de sodium par des composés salins à base de potassium (MARTENS et al., 1976 ; BuckLey et al., 1983).

Cependant, outre sa contribution à la saveur et son rôle bactériostatique, le chlorure de sodium a une influence déterminante par son rôle propre et, de manière indirecte, par son action sur l'activité des enzymes et notamment des protéases. Il en résulte que la substitution d'un autre sel au chlorure de sodium peut nécessiter une adaptation de la technologie de fabrication (MARTENS et al., 1976 ; Karahadian et Lindsay, 1984).

Une revue récente de LINDSAY et al. (1985), fait le point des connaissances actuelles sur les conséquences organoleptiques et technologiques de la fabrication de fromages à teneur réduite en sodium.

Dans le cas du fromage type gruyère (CURTAT, 1982), la suppression totale du salage conduisant à des produits insipides, nous avons envisagé l'utilisation d'une saumure appauvrie en sodium commercialisée par la Société des Salins du Midi et des Salines de l'Est. Cette solution est un sous-produit de l'extraction du sel et a d'ailleurs été utilisée pour d'autres denrées alimentaires à des fins d'assaisonnement (YAMADA, 1981). A la suite des premiers essais un brevet couvrant l'utilisation de cette solution pour la fabrication de fromages hyposodés a été déposé (brevet $\mathrm{n}^{\circ} 84$ 19982). L'objectif fixé dans cette étude était d'obtenir des fromages du type gruyère ayant une teneur réduite en sodium tout en conservant si possible, les qualités organoleptiques propres du produit et la technologie de fabrication.

\section{Matériels et méthodes}

\section{A. Schéma expérimental de fabrication}

Cinq coopératives fromagères ont participé à cette expérimentation. Le lait mis en ouvre était un lait cru entier ou partiellement écrémé provenant du mélange du lait de la traite du soir et de celle du matin de la fabrication. La technologie de transformation, particulière à chacune des coopératives, était comparable à celle décrite par PETER et al. (1953). 
Dans chacun des ateliers ont été fabriquées quatre séries de trois fromages identiques provenant d'une même cuve de fabrication.

Traditionnellement, le salage de ces fromages s'effectue par apport en surface d'une poignée de gros sel. Trois ou quatre heures après, lorsque le sel est fondu, la face salée est frottée à l'aide d'un morceau de toile de jute. Le lendemain, le fromage est retourné pour saler la seconde face. Les faces sont salées et frottées alternativement alors que le talon est soigné à chaque manipulation. Au début, les soins sont quotidiens mais ils deviennent de moins en moins fréquents avec l'implantation des microorganismes qui constituent la "morge ». A un mois les fromages ne sont soignés (salés) que deux fois par semaine et une à deux fois par mois en cave froide.

Dans cette expérimentation, pour une même série, un fromage témoin était salé avec du gros sel, alors que les deux fromages essais étaient frottés selon la même technique, après avoir versé $50 \mathrm{ml}$ d'une solution "Chlorumag 600 » contenant en particulier $600 \mathrm{~g} / \mathrm{l}$ de chlorure de magnésium pour l'un et une solution «Chlorumag 800 », à $800 \mathrm{~g} / \mathrm{l}$ pour l'autre. Durant toute la phase d'affinage, l'ensemble des fromages a été soigné avec la même périodicité.

TABleau 1

Composition type des solutions Chlorumag

Average mineral composition of chlorumag solutions

\begin{tabular}{|c|c|c|c|c|c|c|c|c|c|c|}
\hline \multirow[b]{3}{*}{ Chlorumag 600} & \multicolumn{10}{|c|}{ Sels cristallisés et/ou anhydres (en g/1) } \\
\hline & 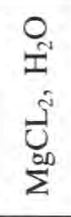 & 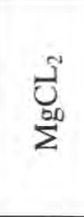 &  & $\begin{array}{l}0^{+} \\
\infty^{+} \\
\sum^{\infty}\end{array}$ & $\begin{array}{l}\overline{\tilde{Z}} \\
\bar{Z}\end{array}$ & $\bar{y}$ & $\overline{0}$ & ${ }_{2}^{\infty}$ & రీ & $\frac{\pi}{2}$ \\
\hline & 600 & 281,2 & 172 & 84 & 28,5 & 27,5 & 239,2 & 147,1 & 55,8 & 6,66 \\
\hline Chlorumag 800 & 800 & 375 & 136 & 66,4 & 10 & 10 & 290,2 & 180,8 & 44,1 & 6,85 \\
\hline
\end{tabular}

Ces solutions, «Chlorumag 600 » et «Chlorumag 800 », respectivement notées $\mathrm{S} 600$ et S800 et dont la composition est donnée dans le tableau 1, sont des sous-produits liquides naturels de l'extraction physique du chlorure de sodium de l'eau de mer.

\section{B. Analyses chimiques}

\section{Echantillonnage}

Les prélèvements faits à mi-rayon sur toute l'épaisseur du fromage, ont été réalisés à l'aide d'un emporte-pièce cylindrique de $20 \mathrm{~mm}$ de diamètre interne. 


\section{Méthodes d'analyses chimiques}

Les analyses ont été faites sur la totalité de l'échantillon après élimination de la croûte (environ $2 \mathrm{~mm}$ à chaque extrémité).

L'Extrait Sec (ES) exprimé en gramme pour $100 \mathrm{~g}$ de fromage est obtenu par dessication à $105^{\circ} \mathrm{C}$ selon les normes d'analyses définies par Serres et al. (1969).

- Le $\mathrm{pH}$ est mesuré avec une électrode de contact au $\mathrm{KCl}$ après broyage du fromage.

- La matière grasse (en gramme pour $100 \mathrm{~g} \mathrm{~d}$ d'ES) est déterminée selon la méthode butyrométrique de Heiss (1962). (1977).

- Le calcium est dosé par complexométrie selon la technique de Pearce

- Les chlorures sont déterminés à l'aide d'un chlorumètre 926 étalonné suivant la norme FIL-IDF, 17 A (1972).

- Les teneurs en sodium, magnésium et potassium sont dosées au spectrophotomètre à flamme sur les cendres du fromage reprises dans du $\mathrm{HCl} 0,01$ $\mathrm{N}$. Les mesures ont été faites à $589 \mathrm{~nm}$ pour le sodium, à $285,2 \mathrm{~nm}$ pour le magnésium et à $766,5 \mathrm{~nm}$ pour le potassium. Le dosage des acides gras volatils a été réalisé par chromatographie en phase gazeuse conformément à la méthode décrite par Berdagué (1986 b).

- L'hydrolyse des caséines a été déterminée par électrophorèse sur gel de polyacrylamide agarose selon la technique d'Uriel (1966) adaptée par Collin et al. (1987).

\section{Analyses sensorielles}

Les qualités organoleptiques et la présentation des fromages ont été jugées à 90 jours.

Le jury était composé par un panel de 15 personnes expérimentées appartenant à la profession fromagère ou au laboratoire INRA de Poligny.

Les évaluations sensorielles sont de type analytique descriptive. Elles s'effectuent sur une tranche de fromage découpée en dés d'environ $0,5 \mathrm{~cm}$ de côté. Ces morceaux étant aléatoirement répartis dans une boîte, le dégustateur qui prenait plusieurs morceaux avait une idée de la qualité de l'ensemble du fromage et attribuait sa note en conséquence. Le jury avait à se prononcer sur l'intensité de quatre critères: la saveur salée, l'arôme caractéristique des fromages du type gruyère (saveur fruitée), l'amertume et la fermeté de la pâte.

La notation était faite sur une échelle allant de 0 à 5 (0: absence ; 1 : très faible; 2 : faible ; 3 : ni faible ni forte ; 4 : forte ; 5 : très forte). En outre, les trois fromages (un témoin et deux essais) étant jugés en même temps, il était demandé aux membres du jury de classer par ordre de préférence les trois échantillons qui leur étaient présentés. 


\section{TABLEAU 2}

Composition chimique des fromages avant salage et, à 3 mois, après salage traditionnel (témoin) ou salage avec les solutions Chlorumag 5600 et 5800 . Les teneurs en minéraux sont exprimées en $\mathrm{mg}$ pour $100 \mathrm{~g}$ de fromage. Comparaison par rapport aux témoins : différence non significative $(N S)$, significative à $5 \%$ ou $1 \%{ }^{* *}$. Moyennes $(\bar{x})$ et écart-types(s)

Chemical composition of cheese before salting and after 3 months of traditional (reference) salting or salting with S600 and S800 Chlorumag solutions. The mineral content is expressed in $\mathrm{mg} / 100 \mathrm{~g}$ of cheese. Comparisons with the reference : non significant (NS), significant $5 \% *, 1 \% * *$ Mean $(\bar{x})$ and standard deviation $(s)$

\begin{tabular}{|c|c|c|c|c|c|c|c|c|c|}
\hline \multirow{3}{*}{ Variables } & \multirow{2}{*}{\multicolumn{2}{|c|}{$\begin{array}{l}\text { Avant traitement } \\
\text { Before salting }\end{array}$}} & \multicolumn{6}{|c|}{$\begin{array}{l}\text { Après salage } \\
\text { After salting }\end{array}$} & \multirow{3}{*}{$\begin{array}{l}\text { Test } \\
\text { par paires } \\
\text { Paired test }\end{array}$} \\
\hline & & & \multicolumn{2}{|c|}{$\begin{array}{l}\text { Témoin } \\
\text { Reference }\end{array}$} & \multicolumn{2}{|c|}{ S600 } & \multicolumn{2}{|c|}{ S800 } & \\
\hline & $\overline{\mathrm{x}}$ & $\mathrm{s}$ & $\overline{\mathrm{x}}$ & $\mathrm{s}$ & $\overline{\mathrm{x}}$ & $\mathrm{s}$ & $\overline{\mathrm{x}}$ & $\mathrm{s}$ & \\
\hline $\mathrm{pH}$ & 5,35 & 0,06 & 5,72 & 0,07 & 5,68 & 0,07 & 5,67 & 0,07 & $* *$ \\
\hline $\begin{array}{l}\text { Extrait sec }(\%) \\
\text { Dry matter }\end{array}$ & 62,67 & 0,99 & 64,77 & 1,05 & 64,77 & 1,02 & 64,83 & 1,04 & NS \\
\hline $\begin{array}{l}\text { Matière grasse } \\
\text { Fat }(\%)\end{array}$ & - & - & 49,83 & 1,74 & 49,88 & 1,74 & 49,86 & 1,69 & NS \\
\hline $\mathrm{Cl}$ & - & - & 700,65 & 123,13 & 333 & 76,97 & 381,3 & 77,18 & $* * *$ \\
\hline $\mathrm{Ca}$ & 964 & 30,73 & 953,3 & 38,50 & 987,0 & 33,93 & 991,4 & 43,32 & $* *$ \\
\hline $\mathrm{Na}$ & 35 & 2,41 & 272,0 & 44,76 & 46,2 & 5,55 & 44,9 & 5,05 & $* *$ \\
\hline $\mathrm{Mg}$ & 33 & 1,22 & 33,2 & 1,57 & 72,8 & 11,47 & 78,85 & 13,51 & $* *$ \\
\hline K & 108 & 6,49 & 102,2 & 9,4 & 109,1 & 5,57 & 105,85 & 4,9 & NS \\
\hline
\end{tabular}




\section{Résultats}

\section{A. Résultats analytiques}

\section{Caractéristiques physico-chimiques}

L'ensemble des résultats est rassemblé dans le tableau 2. On constate que la substitution des solutions "Chlorumag " au chlorure de sodium abaisse légèrement le $\mathrm{pH}$ en fin d'affinage mais ne modifie pas la teneur en extrait sec.

En ce qui concerne les teneurs en minéraux, seule la teneur en potassium n'est pas influencée par le traitement.

Par rapport au salage traditionnel, l'utilisation des solutions de magnésium permet de réduire d'au moins $80 \%$ la teneur en sodium des fromages, soit 50 $\mathrm{mg}$ en moyenne au lieu de $270 \mathrm{mg}$ pour $100 \mathrm{~g}$ de fromage. Si l'on tient compte de la composition initiale moyenne de l'ensemble des fromages avant le début des soins (tabl. 2) l'apport de sodium réalisé avec l'une ou l'autre des solutions de substitution avoisine $10 \mathrm{mg}$.

En outre, les solutions S600 et S800 utilisées conduisent à une augmentation sensible de la teneur en magnésium des fromages soit respectivement $+39,6 \mathrm{mg}(+119 \%)$ et $+45,6 \mathrm{mg}(+137 \%)$. Il en est de même de la teneur en calcium qui augmente de $+34 \mathrm{mg}(3,55 \%)$ et $+38 \mathrm{mg}(4,0 \%)$.

Si l'apport de magnésium est dû à la nature des solutions employées, il n'est est pas de même pour le calcium puisque ces solutions n'en contiennent pas. Cette augmentation de la teneur en calcium dans la pâte des fromages ne peut s'expliquer que par une moindre migration de cet élément vers la croûte qui a été éliminée avant analyse.

Cette moindre teneur en calcium des fromages témoins corrobore les observations faites par Berdagué (1986a) sur le Comté.

\section{Profils fermentaires et protéolyse}

Le tableau 3 montre que l'apport de magnésium ne modifie pas les phénomènes fermentaires et protéolytiques qui se passent durant les trois mois d'affinage. Excepté un léger effet activateur du magnésium sur l'hydrolyse de la caséine $\alpha \mathrm{S} 1$, il n'existe pas de différence significative entre les fromages d'une même série. Il apparaît donc que le magnésium se substituerait au sodium comme facteur limitant la protéolyse. En effet, dans les essais de réduction de la teneur en sodium sans subtitution par d'autres éléments, plusieurs auteurs (DelbeKe et al., 1982; ThaKur et al., 1979 et BurCKLEY et Fitzegerald, 1983), ont observé un net accroissement de la protéolyse et de sa cinétique. 


\section{TABLEAU 3}

Influence du type de salage sur la protéolyse et la teneur en acides gras volatils (AGV) des fromages: comparaison par rapport au témoin (NS: non significatif ; * significative $5 \%$

- caséines: Moyennes ( $\bar{x})$ et écart-type(s) des pourcentages des différentes fractions protéiques insolubles à $\mathrm{pH}$ 4,6 mesurées par électrophorèse en gel de polyacrylamide.

$\beta$ deg : fragment de caséine $\beta$ non identifié.

- AGV: Moyennes $(\bar{x})$ et écart-types $(s)$ des teneurs en acides gras volatils exprimées en $\mathrm{mg} / 100 \mathrm{~g}$.

Influence of the kind of salting on cheese proteolysis and volatile fatty acid content:

Comparison with the reference : NS: non significant; * significant $5 \%$.

- Caseins: Means $(\bar{x})$ and standard deviation $(s)$ of the various pH 4.6 insoluble protein fractions, measured by electrophoresis (PAGE).

$\beta$ deg : unidentified $\beta$ casein fragment.

- AGV: Means $(\bar{x})$ and standard deviation(s) of the volatile fatty acid content of cheese in $\mathrm{mg} / 100 \mathrm{~g}$.

\begin{tabular}{|c|c|c|c|c|c|c|c|c|}
\hline & \multirow{2}{*}{ Variables } & \multicolumn{2}{|c|}{$\begin{array}{l}\text { Témoin } \\
\text { Reference }\end{array}$} & \multicolumn{2}{|c|}{ Chlorumag S600 } & \multicolumn{2}{|c|}{ Chlorumag S800 } & \multirow{2}{*}{$\begin{array}{c}\text { Test } t \\
\text { par } \\
\text { paires } \\
\text { Paired } \\
\text { t test }\end{array}$} \\
\hline & & $\overline{\mathrm{x}}$ & $\mathrm{s}$ & $\overline{\mathrm{x}}$ & $\mathrm{s}$ & $\overline{\mathrm{x}}$ & $\mathrm{s}$ & \\
\hline \multirow{8}{*}{ 这 } & Para $\kappa$ & 6,8 & 2,06 & 6,2 & 2,67 & 6,1 & 2,05 & NS \\
\hline & $\beta$ deg. & 2,8 & 0,56 & 2,9 & 0,90 & 3,0 & 0,46 & NS \\
\hline & $\gamma 1$ & 8,5 & 0,62 & 8,6 & 0,56 & 8,4 & 0,70 & NS \\
\hline & $\gamma 2$ & 6,8 & 0,61 & 7,0 & 0,51 & 6,7 & 0,59 & NS \\
\hline & $\gamma 3$ & 6,8 & 0,75 & 6,9 & 0,69 & 6,6 & 0,61 & NS \\
\hline & $\beta$ & 18,8 & 1,34 & 19,6 & 1,63 & 19,6 & 1,67 & NS \\
\hline & $\alpha \mathrm{SI}$ & 16,7 & 1,53 & 15,2 & 11,56 & 15,3 & 1,99 & * \\
\hline & $\alpha$ SII & 25,4 & 1,62 & 26,4 & 0,86 & 27,0 & 1,27 & * \\
\hline \multirow{4}{*}{$\begin{array}{l}> \\
0 \\
\dot{4}\end{array}$} & $\mathrm{C} 2$ & 220,2 & 145,0 & 195,2 & 123,49 & 195,0 & 88,92 & NS \\
\hline & $\mathrm{C} 3$ & 88,1 & 68,26 & 105,5 & 91,72 & 128,9 & 126,36 & NS \\
\hline & $\mathrm{C} 4$ & 8,4 & 5,72 & 8,3 & 3,54 & 9,6 & 7,31 & NS \\
\hline & iC5 & 4,2 & 4,67 & 3,5 & 2,31 & 3,0 & 1,27 & NS \\
\hline
\end{tabular}

B. Présentation des fromages

\section{Le croûtage}

L'utilisation des solutions "Chlorumag " semble modifier la nature des micro-organismes de la morge. La morge qui est habituellement de couleur gris jaune présente, après traitement avec les solutions de Chlorumag, une nette coloration rougeâtre dont l'intensité croît avec la concentration en magnésium. Au cours du stockage en chambre froide, alors que les soins sont plus espacés, cette coloration s'estompe. 
TABLEAU 4

Notes des caractéristiques sensorielles des fromages en fonction du type de salage. Moyennes $(\bar{x})$ et écart-types(s)

Scores of the sensory characteristics of cheeses according to the type of salting. Mean $(\bar{x})$ and standard deviation(s)

\begin{tabular}{|c|c|c|c|c|c|c|c|c|c|c|c|c|}
\hline \multirow{3}{*}{ Variable } & \multicolumn{12}{|c|}{$\begin{array}{l}\text { Type de salage } \\
\text { Type of salting }\end{array}$} \\
\hline & \multicolumn{4}{|c|}{$\begin{array}{l}\text { Témoin } \\
\text { Reference }\end{array}$} & \multicolumn{4}{|c|}{ CHLORUMAG S600 } & \multicolumn{4}{|c|}{ CHLORUMAG S800 } \\
\hline & $\overline{\mathrm{x}}$ & $\operatorname{mini}$ & $\operatorname{maxi}$ & $\mathrm{s}$ & $\overline{\mathrm{x}}$ & $\operatorname{mini}$ & $\operatorname{maxi}$ & s & $\overline{\mathrm{x}}$ & $\operatorname{mini}$ & $\operatorname{maxi}$ & s \\
\hline $\begin{array}{l}\text { Salinité } \\
\text { Saltiness }\end{array}$ & 2,71 & 2,2 & 3,21 & 0,28 & 1,43 & 0,78 & 2,0 & 0,30 & 1,46 & 1,06 & 1,86 & 0,19 \\
\hline $\begin{array}{l}\text { Fruité } \\
\text { Flavour intensity }\end{array}$ & 2,72 & 2,0 & 3,31 & 0,38 & 1,76 & 1,0 & 2,29 & 0,38 & 1,54 & 0,81 & 2,29 & 0,37 \\
\hline $\begin{array}{l}\text { Dureté } \\
\text { Firmness }\end{array}$ & 3,09 & 2,27 & 4,19 & 0,44 & 2,47 & 1,71 & 3,50 & 0,40 & 2,74 & 2,06 & 3,63 & 0,48 \\
\hline
\end{tabular}




\section{L'ouverture}

Les fromages ont été coupés en deux afin d'apprécier l'incidence de la teneur en sodium sur l'ouverture (répartition, taille et nombre).

Lorsque les fromages témoins ne présentent pas de défaut d'ouverture, on constate que l'utilisation des solutions magnésiennes ne modifie pas le développement de l'ouverture. Par contre, lorsque le témoin présente un excès d'ouverture, les solutions employées pour les deux autres fromages amplifient le défaut. Celui-ci est d'autant plus prononcé que la solution est plus concentrée en magnésium.

\section{Caractéristiques sensorielles}

Les résultats moyens des analyses sensorielles des 16 séries de fromages sont donnés dans le tableau 4.

On constate que les fromages soignés traditionnellement sont plus fermes, plus salés et plus fruités que les fromages essais dont le caractère amer est perceptible. Soulignons que les moyennes des notes d'amertume des fromages traités avec $S 600$ et $S 800(1,99$ et 2,34$)$ indiquent une amertume faible, contre très faible pour les témoins $(0,90)$, les notes d'amertume ne dépassant jamais l'échelle 3 (ni faible ni forte). Quant au caractère fruité, les fromages témoins avec une note moyenne de 2,72 ne représentaient pas des fromages caractéristiques d'un bon Comté. Bien que significativement différentes, les notes moyennes des essais ne traduisent pas une différence très grande $(1,76$ à 1,54$)$.

Les fromages traités avec la solution S600 sont moins amers, moins fermes et légèrement plus fruités que ceux traités avec la solution S800. Par contre, dans ces deux cas, la perception de la salinité est comparable. Il est à noter que le caractère fruité est fortement corrélé avec l'amertume décelée dans ces fromages $(\mathrm{r}=-0,855)$.

Les analyses de variance effectuées sur les critères retenus pour l'appréciation des fromages montrent (tabl. 4) que ces caractéristiques organoleptiques dépendent à la fois de l'origine des fromages et du traitement mais qu'il n'existe pas d'interaction significative entre les deux facteurs. Ces résultats traduisent, au niveau des caractéristiques sensorielles des différences parfois aussi importantes entre fromageries qu'entre traitement et témoin.

Le jury ayant classé les 3 fromages d'une même série par ordre de préférence, on a réalisé une analyse de variance sur les rangs (test de Friedman). Bien que pour toutes les séances de dégustation le fromage témoin ait été le plus apprécié devant les deux essais, ce classement n'avait une signification que onze fois sur seize.

\section{Relations entre les caractéristiques sensorielles et les caractéristiques physico-chimiques}

L'aspect gustatif le plus important de cette expérimentation réside dans la légère augmentation de la saveur amère dans les fromages essais.

L'amertume décelée dans ces fromages peut avoir deux origines : 
- la protéolyse des caséines $\beta$ en caséines $\gamma$ génératrices du goût amer (DELBeKe et al., 1982) ou plus encore en peptides formés de la lyse de la partie C terminale (VISSER et al., 1983) Dans ce cas la perception de l'amertume est liée à la concentration de cette fraction peptidique ;

- la teneur en magnésium des fromages.

TABLEAu 5

Pourcentage des variances des différentes caractéristiques sensorielles des fromages expliquées par les facteurs fruitières (5 fruitières)

et type de salage (3 traitements).

Influence du facteur : NS : non significative ; ${ }^{*}$ : significative $5 \% ;{ }^{* *}$ : significative $1 \%$.

Percent of the variances of the various sensory properties of cheese explained

by the cheese origin (5 cheese plants) and type of salting (3 treatments).

Influence of factors : NS : non significant ; ${ }^{*}$ : significant $5 \%$; ${ }^{* *}$ : significant $1 \%$.

\begin{tabular}{|c|c|c|c|c|}
\hline \multirow{2}{*}{ Variable } & \multicolumn{4}{|c|}{$\begin{array}{l}\text { Facteurs } \\
\text { Factors }\end{array}$} \\
\hline & $\begin{array}{l}\text { Fruitière } \\
\text { Cheese plant }\end{array}$ & $\begin{array}{l}\text { Salage } \\
\text { Salting }\end{array}$ & Interaction & Total \\
\hline $\begin{array}{l}\text { Salinité } \\
\text { Saltiness }\end{array}$ & $6,0 * *$ & 82,1 ** & NS 3,01 & 91,1 \\
\hline $\begin{array}{l}\text { Amertume } \\
\text { Bitterness }\end{array}$ & $6,2 *$ & $77,5 * *$ & NS 3,01 & 86,7 \\
\hline $\begin{array}{l}\text { Fruité } \\
\text { Flavour intensity }\end{array}$ & $15,6^{*}$ & $63,1 * *$ & NS $\quad 2,6$ & 81,3 \\
\hline $\begin{array}{l}\text { Dureté } \\
\text { Firmness }\end{array}$ & $18,9 *$ & 30,6 ** & NS 12,9 & 62,4 \\
\hline
\end{tabular}

En l'absence de toute analyse fine de la fraction azotée, nous ne sommes pas en mesure de définir l'importance relative de ces deux sources d'amertume. Toutefois, la présence de peptides amers ne peut pas être totalement écartée puisque l'on constate d'une part, une très faible amertume dans les fromages témoins et d'autre part, il a été montré que des teneurs élevées en sel dans les fromages réduisaient la formation de peptides amers (STADHOUDERS et al., 1983). Le fait que l'on ait fortement réduit la teneur en sel des fromages essais pourrait avoir favorisé ce caractère.

Si l'on considère cette fois l'influence de la teneur en matière grasse, des auteurs (BERDAGué, 1986a ; ThAKur et al., 1975) ont observé une corrélation négative entre l'amertume et la teneur en matière grasse. En revanche, les résultats organoleptiques de notre étude indiquent que l'intensité de l'amertume n'est pas liée à la teneur en matière grasse (tabl. 6) ; on constate même que les fromages les plus gras sont également les plus amers. Par contre, ils renferment plus de magnésium. Ce dernier résultat confirme l'hypothèse selon laquelle la teneur en magnésium constituerait le principal facteur de l'augmentation de la saveur amère puisque la teneur en magnésium et la note d'amertume sont très corrélées $(\mathrm{r}=0,71)$. 


\section{TABleau 6}

Comparaison de la composition physico-chimique et de l'amertume des fromages classés en fonction de leur teneur en matière grasse.

$\bar{x}$ : moyenne; $s$ : écart-type

Groupe I: teneur normale en matière grasse

Groupe II: teneur élevée en matière grasse

Différence entre les 2 groupes: * significative à $5 \%$; ** significative à $1 \%$;

NS: non significative

Comparison of the physico-chemical characteristic and bitterness intensity of normal fat (groupe I) and high fat (groupe II) cheeses.

$\bar{x}:$ mean; $s:$ standard deviation

Difference between the two groups: NS non significant; * significant $5 \%$; ** significant $1 \%$.

\begin{tabular}{|c|c|c|c|c|c|}
\hline & \multicolumn{2}{|c|}{$\begin{array}{l}\text { Groupe I } \\
(\mathrm{n}=28)\end{array}$} & \multicolumn{2}{|c|}{$\begin{array}{c}\text { Groupe II } \\
(\mathrm{n}=12)\end{array}$} & \multirow{2}{*}{$\begin{array}{c}\text { Significatior } \\
\text { test } \mathrm{t} \\
t \text { test }\end{array}$} \\
\hline & $\overline{\mathrm{x}}$ & $\mathrm{s}$ & $\overline{\mathrm{x}}$ & $\mathrm{s}$ & \\
\hline $\begin{array}{l}\text { E.S. } \\
\text { Dry matter }\end{array}$ & 64,40 & 0,94 & 65,72 & 0,42 & $* *$ \\
\hline $\begin{array}{l}\text { Matière grasse } \quad \% \\
\text { Fat }\end{array}$ & 48,89 & 0,56 & 52,45 & 0,65 & $* *$ \\
\hline $\mathrm{Mg}_{\mathrm{mg} / 100 \mathrm{~g}}$ & 72,64 & 9,71 & 78,70 & 17,43 & NS \\
\hline $\mathrm{Na} \mathrm{mg} / 100 \mathrm{~g}$ & 46,00 & 6,12 & 46,00 & 5,33 & NS \\
\hline $\begin{array}{l}\text { Amertume } \\
\text { Bitterness }\end{array}$ & 2,07 & 0,37 & 2,37 & 0,41 & * \\
\hline
\end{tabular}

D'après ces résultats, il semble bien que la sensation d'amertume correspondrait plutôt à une perception autre que celle d'amertume mais qu'il est difficile de caractériser. Ce goût particulier dont l'intensité est liée à la teneur en magnésium peut être assimilé à ce que MARTENS et al. (1978) qualifiaient de "goût de potassium » pour le Gouda hyposodé obtenu en substituant le chlorure de potassium au chlorure de sodium.

Le deuxième fait marquant de l'emploi de ces saumures est la diminution de la fermeté de la pâte. Ce caractère sensoriel est corrélé à la teneur en sodium du fromage comme l'ont également montré THAKur et al. (1975), Martens et al. (1976) et Berdagué (1986a). De Jong (1976) a montré qu'il existait une relation entre la dégradation des caséines $\alpha \mathrm{S} 1$ et la diminution de la fermeté dans le cas des pâtes molles. Il semble qu'il existerait une telle relation dans le cas de cette étude. 


\section{Conclusion}

L'utilisation de ces solutions sous produit de l'extraction du sel de mer et concentrées en chlorure de magnésium, comme substitut au chlorure de sodium permet d'obtenir des fromages du type gruyère ayant une teneur en sodium réduite, inférieure à $50 \mathrm{mg} / 100 \mathrm{~g}$ de fromage sans nuire, d'une façon marquée aux qualités organoleptiques des fromages. A cela, il faut ajouter un enrichissement du produit en calcium et surtout en magnésium.

Reçu le 9 juillet 1986.

Accepté pour publication le 22 décembre 1986.

\section{Remerciements}

Nous remercions Messieurs P. RouSSEAUX et G. MocQuot pour les conseils qu'ils nous ont donnés pour la réalisation de ce travail ainsi que les Etablissements GRILLOT à Poligny qui ont assuré l'affinage des fromages.

\section{Références bibliographiques}

Berdagué J.L., 1986a. Affinage du Gruyère de Comté. Thèse de doctorat de l'Université de Dijon.

Berdagué J.L., 1986b. Méthode rapide d'extraction des acides gras volatils des fromages. Lait, 66 , 233-246.

Buckley J. Fitzegerald E., 1983. Effect of total and partial substitution of sodium chloride on the quality of Cheddar cheese. Food Sci. Technol. Abstr., 1, 145-146.

Collin J.C., Berdagué J.L., Dognin-Bergeret M., Grappin R., 1987. Affinage du Gruyère de Comté : étude de la protéolyse. Lait, 67 (3), 299-318.

Curtat G.E., 1982. Fromage de Gruyère, notamment Comté, et son procédé de fabrication. Brevet français $\mathrm{n}^{\circ} 8201365$.

Curtat G.E., 1984. Procédé de fabrication d'un fromage hyposodé, notamment de fromage de gruyère hyposodé. Brevet français $\mathrm{n}^{\circ} 8419382$.

De Jong L., 1976. Protein breakdown in soft cheese and its relation to consistency. I. Proteolysis and consistency of Noordhollandse Meshanger's cheese. Neth. Milk Dairy J., 30, 242-253.

Delbeke R., Paelinck H., Martens R., 1982. La fabrication du fromage type Gouda à faible teneur en matière grasse et en sodium. Rev. Agric., 35, 2717-2735.

Heiss E., 1961. Essais de dosage de la matière grasse dans le fromage par des méthodes rapides. Dtsch. Molk. Ztg., 82, 3.

Karahadjan C., Lindsay R.C., 1984. Flavor and textural properties of reduced sodium process american cheese. J. Dairy Sci., 67, 1892-1904.

Lindsay R.C., Karahadjan C., Amundson C.H., 1985. Low sodium cheese : overview and properties of cheddar cheese made with U.F. and R.O. retentate supplemented milk. In : Proceedings of IDF Seminar. Atlanta Ga. USA, ed., FIL-IDF, Bruxelles, 55-76.

Martens R.R., Vanderpoorten R., Naudts M., 1976. Fabrication, composition et propriétés du fromage Gouda à teneur réduite en sodium. Rev. Agric., 29, 681-697. 
Pearce K.N., 1977. The complexometric determination of calcium in dairy products. N.Z. J. Dairy Sci. Technol., 12, 113-115.

Peter A., Zollikofer E., Badoux F., 1953. Manuel de la fabrication du fromage d'Emmental. Editions des Hoirs, Berne.

Serres L., Amariglio S., Petransxiene D., 1969. Contrôle de la qualité des produits laitiers. Tome 1. Document Ministère de l'Agriculture.

Stadhouders J., Hup G., Exterkate A.F., Visser S., 1983. Bitter flavour in cheese. 1. Mechanism of the formation of the bitter flavour defect in cheese. Neth. Milk Dairy J., 37, 157-167.

Thakur M.K., Kirk J.R., Hedrick T.I., 1975. Changes during ripening of unsalted Cheddar cheese. J. Dairy Sci., 58, 175-180.

Visser S., Slangen K.J., Hup G., Stadhouders J., 1983. Bitter flavour in cheese. 3. Comparative gel-chromatographic analysis in hydrophobic peptide fractions from twelve Gouda-type cheese and identification of bitter peptides isolated from a cheese made with Streptococcus cremoris strain HP1.2. Neth. Milk Dairy J., 37, 181-192.

YAMADA J.P., 1981. Composition d'assaisonnement. Brevet français nº 8120849. 